\title{
Clinical Significance of the Champagne Bottle Neck Sign in the Extracranial Carotid Arteries of Patients with Moyamoya Disease
}

\author{
(D). Yasuda, (DS. Arakawa, (D). Shimogawa, (D). Kanazawa, (D). Sayama, (D). Haga, and (D). Morioka
}

\begin{abstract}
BACKGROUND AND PURPOSE: The champagne bottle neck sign represents a rapid reduction in the extracranial ICA diameters and is a characteristic feature of Moyamoya disease. However, the clinical significance of the champagne bottle neck sign is unclear. We investigated the relationship between the champagne bottle neck sign and the clinical and hemodynamic stages of Moyamoya disease.
\end{abstract}

MATERIALS AND METHODS: We analyzed 14 patients with Moyamoya disease before revascularization ( 5 men, 9 women; age, $43.2 \pm 19.3$ years). The ratio of the extracranial ICA and common carotid artery diameters was determined using carotid ultrasonography or cerebral angiography; a ratio of $<0.5$ was considered champagne bottle neck sign-positive. The clinical disease stage was determined using the Suzuki angiographic grading system. CBF and cerebral vasoreactivity also were measured.

RESULTS: The ICA/common carotid artery ratio (expressed as median [interquartile range]) decreased as the clinical stage advanced (stages I-II, 0.71 [0.60-0.77]; stages III-IV, 0.49 [0.45-0.57]; stages V-VI, 0.38 [0.34-0.47]; $P<.001$ ). Lower ICA/common carotid artery ratio tended to occur in symptomatic versus asymptomatic arteries $(0.47$ [0.40-0.53] versus 0.57 [0.40-0.66], respectively; $P=.06)$. Although the ICA/common carotid artery ratio was not related to cerebral perfusion, it decreased as cerebral vasoreactivity decreased ( $P<.01)$. All champagne bottle neck sign-positive arteries were classified as Suzuki stage $\geq$ III, $73 \%$ were symptomatic, and $89 \%$ exhibited reduced cerebral vasoreactivity. In contrast, all champagne bottle neck sign-negative arteries were Suzuki stage $\leq$ III, $67 \%$ were asymptomatic, and all showed preserved cerebral vasoreactivity.

CONCLUSIONS: The champagne bottle neck sign was related to advanced clinical stage, clinical symptoms, and impaired cerebral vasoreactivity. Thus, detection of the champagne bottle neck sign might be useful in determining the clinical and hemodynamic stages of Moyamoya disease.

ABBREVIATIONS: CBNS = champagne bottle neck sign; CCA = common carotid artery; CVR = cerebral vasoreactivity; MMD = Moyamoya disease

M oyamoya disease (MMD) is a cerebrovascular disorder characterized by progressive bilateral stenosis or occlusion of the distal portion of the ICA and the proximal portion of the MCAs and anterior cerebral arteries. Affected patients also have an abnormal vascular network (Moyamoya vessels) at the base of

Received December 16, 2015; accepted after revision March 18, 2016.

From the Departments of Cerebrovascular Disease (C.Y., S.A., Y.K.) and Neurosurgery (T.Shimogawa, T.Sayama, S.H., T.M.), Japan Labour Health and Welfare Organization, Kyushu Rosai Hospital, Kitakyushu, Japan.

Authors' contributions: Literature search, figures, study design, data collection, data analysis, data interpretation, writing (C.Y.); literature search, study design, data analysis, data interpretation, writing (S.A.); data interpretation (T.Shimogawa, T.Sayama, S.H.); literature search, data interpretation (Y.K.); study design, data interpretation, writing (T.M.).

Please address correspondence to Chiharu Yasuda, MD, Department of Neurology, University of Occupational and Environmental Health of Japan, Wakamatsu Hospital, 1-17-1 Hamamachi Wakamatsu-ku, Kitakyushu 808-0024, Japan; e-mail: akaike_komachi@yahoo.co.jp

http://dx.doi.org/10.3174/ajnr.A4815 the brain. The vascular stenosis extends to the extracranial ICA as the disease advances. ${ }^{1}$ In 1997 , Yang et $\mathrm{al}^{2}$ used angiography to demonstrate stenotic lesions of the extracranial ICA in $60 \%$ of patients with MMD. In 2006, Yasaka et $\mathrm{al}^{3}$ reported that carotid ultrasonography showed rapid reduction of the diameter at the proximal portion of the ICA, revealing the appearance of a champagne bottle neck in a patient with MMD. The champagne bottle neck sign (CBNS), which is easily detected noninvasively via carotid ultrasonography, is an important morphologic feature of the extracranial ICA of patients with MMD. ${ }^{3-5}$ Although the CBNS is reportedly present in $74 \%$ of patients with $\mathrm{MMD},{ }^{3}$ the time at which the CBNS begins to appear during the course of MMD is unclear. In addition, whether the CBNS is related to the clinical or hemodynamic stage of MMD is unknown. Therefore, we investigated the relationship between the appearance of the CBNS and the clinical stage, presence of clinical symptoms, and hemodynamic stage in patients with MMD. 


\section{MATERIALS AND METHODS}

\section{Patients}

We retrospectively evaluated 24 patients newly diagnosed with MMD according to the guidelines proposed by the Ministry of Health, Labour and Welfare of Japan ${ }^{6}$ at our hospital from April 2007 to October 2012. The inclusion criteria were evaluation of the patient before revascularization, evaluation of the extracranial ICA via carotid ultrasonography or DSA, and determination of the severity of MMD via DSA. These criteria excluded 10 patients because DSA was not performed $(n=5)$ or extracranial ICA data were not available $(n=5)$. Therefore, 14 patients with 27 affected arteries were enrolled (bilateral MMD: 13 patients; unilateral MMD: 1 patient). This study was approved by our institutional review committee.

\section{Evaluation of the Extracranial ICA}

The ratio of the ICA diameter (below the mandibular bone) to the common carotid artery (CCA) diameter (proximal aspect to bulbus) was measured via carotid ultrasonography. Arteries with a ratio of $<0.5$ were defined as CBNS-positive. ${ }^{3}$ In 7 arteries, the extracranial ICA diameter could not be adequately estimated via carotid ultrasonography because of the high position of the carotid bifurcation. Therefore, the DSA findings were used to obtain the ICA/CCA ratio in these arteries. Evaluation of this sign was based on visual inspection by 2 experienced neuroradiologists in our hospital who were blinded to the clinical and imaging data. No differences in the radiologists' interpretations were noted on independent assessments.

\section{Clinical Stage}

The clinical stage of MMD was determined according to the Suzuki angiographic grading system ${ }^{1}$ as follows: In stage I, the carotid fork is narrowed with no other abnormalities. In stage II, the intracerebral main arteries are dilated because of stenosis at the terminal portion of the ICA. In stage III, the MCAs and anterior cerebral arteries are narrowed, and the basal Moyamoya is intensified. In stage IV, the occlusion of the ICA extends to the junction of the posterior communicating artery, resulting in enlargement of the intraorbital Moyamoya vessels and collateral vessels from the external carotid artery. In stage $\mathrm{V}$, the basal Moyamoya vessels shrink, and the MCAs and anterior cerebral arteries disappear; occlusion of the ICA extends as far as C2 or above C3. In stage VI, the siphon of the ICA completely disappears.

\section{Clinical Symptoms}

Clinical symptoms at diagnosis were investigated. The ICA that was responsible for symptoms was defined as symptomatic, and the contralateral side of the ICA was defined as asymptomatic. In incidentally diagnosed cases, the bilateral ICAs were defined as asymptomatic.

\section{SPECT}

$\mathrm{CBF}$ and cerebral vasoreactivity (CVR) to acetazolamide in the MCA territory were measured by SPECT. ${ }^{7-9}$ In this study, 6 patients (12 arteries) who underwent semiquantitative SPECT with iodine $123 \mathrm{~N}$-isopropyl-p-iodoamphetamine were included to evaluate the relationship between the appearance of the CBNS

\begin{tabular}{|c|c|}
\hline Characteristic & \\
\hline Patients (no.) & 14 \\
\hline Age (mean $\pm S D$, range) $(y)$ & $43.2 \pm 19.3,6-71$ \\
\hline \multicolumn{2}{|l|}{ Sex (no.) } \\
\hline Male & 5 \\
\hline Female & 9 \\
\hline \multicolumn{2}{|l|}{ Clinical diagnoses at onset (no.) } \\
\hline Hemorrhagic stroke & 4 \\
\hline Ischemic stroke & 5 \\
\hline Transient ischemic attack & 4 \\
\hline Asymptomatic & 1 \\
\hline Arteries (no.) & 27 \\
\hline \multicolumn{2}{|l|}{ Suzuki grades (no. of arteries) } \\
\hline Stage I & 3 \\
\hline Stage II & 2 \\
\hline Stage III & 12 \\
\hline Stage IV & 4 \\
\hline Stage V & 3 \\
\hline Stage VI & 3 \\
\hline \multicolumn{2}{|c|}{ Evaluation of ICA/CCA ratio (no. of arteries) } \\
\hline Ultrasonography & 20 \\
\hline DSA & 7 \\
\hline \multicolumn{2}{|l|}{ CBNS (no. of arteries) } \\
\hline Positive & 15 \\
\hline Negative & 12 \\
\hline
\end{tabular}

and impairment of cerebral hemodynamics. CBF was semiquantitatively measured before and 15 minutes after intravenous injection of $10 \mathrm{mg} / \mathrm{kg}$ acetazolamide on separate days, with an interval of 2-3 days. ROIs were placed automatically on the bilateral MCA territories with commercially available software (GMS7700R, E.CAM Signature; Toshiba Medical Systems, Tokyo, Japan). The CBF in the MCA territory was obtained, excluding ischemic or hemorrhagic lesions. The CVR to acetazolamide was calculated as follows:

$$
\mathrm{CVR}=100 \times\left(\mathrm{CBF}_{\mathrm{ACZ}}-\mathrm{CBF}_{\text {rest }}\right) / \mathrm{CBF}_{\text {rest }},
$$

where $\mathrm{CVR}$ is expressed as a percentage and $\mathrm{CBF}_{\text {rest }}$ and $\mathrm{CBF}_{\mathrm{ACZ}}$ represent $\mathrm{CBF}$ before and after intravenous injection of acetazolamide, respectively. CVR of $<20 \%$ was defined as reduced CVR. ${ }^{8,9}$

\section{Data Analysis}

The relationships between the ICA/CCA ratio (ie, the CBNS) and Suzuki grade, the presence of clinical symptoms, and impairment of cerebral hemodynamics were investigated. Data are expressed as median (interquartile range). Differences in the ICA/CCA ratio among clinical stages and between symptomatic and asymptomatic arteries were examined by the Kruskal-Wallis test followed by the Bonferroni multiple comparison and the Mann-Whitney $U$ test, respectively. Correlation of the ICA/CCA ratio and CVR were evaluated by using the Pearson correlation coefficient. The level of statistical significance was set at $P<.05$. All analyses were performed with JMP 10 software (SAS Institute, Cary, North Carolina).

\section{RESULTS}

We analyzed 27 arteries of 14 patients ( 5 men, 9 women [mean age, $43.2 \pm 19.3$ years; age range, 6-71 years]). Their clinical 

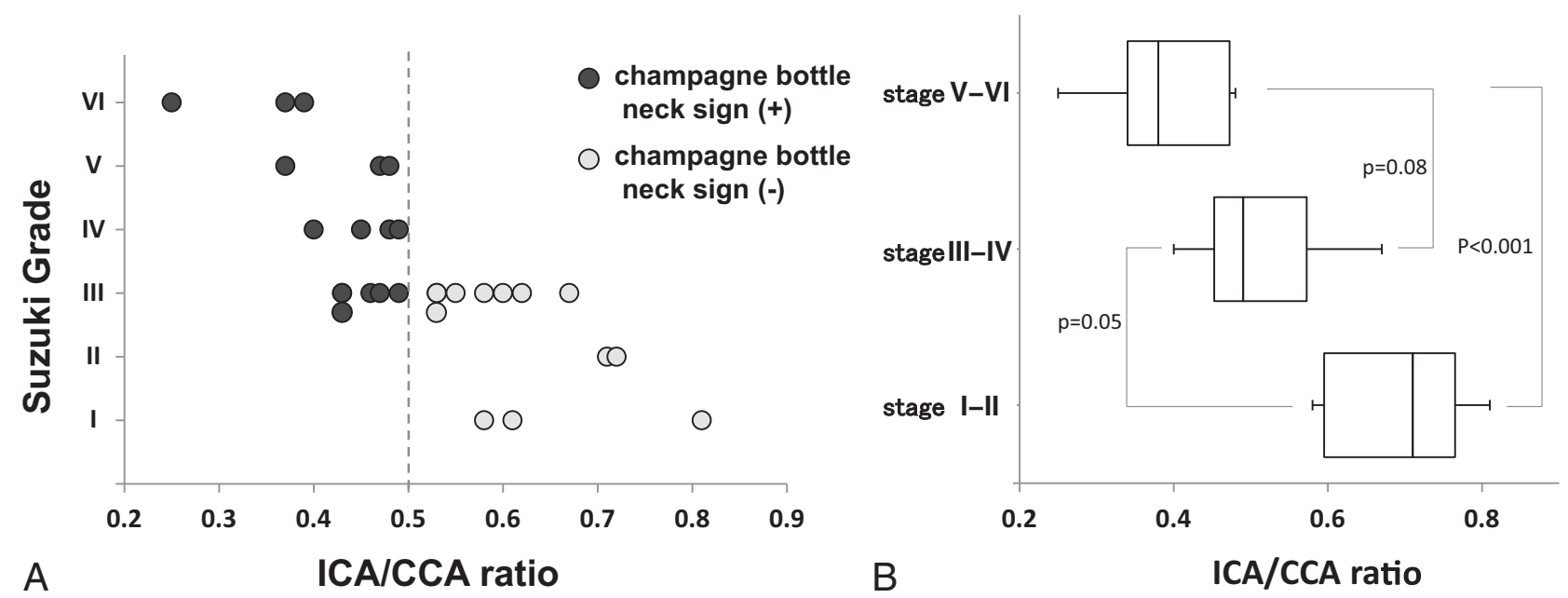

FIG 1. Relationship between the ICA/CCA ratio and the clinical stage (Suzuki stage, I-VI [early-advanced]). A, The ICA/CCA ratio decreased as the clinical stage advanced, and the CBNS was observed in stage III or higher. B, The median ICA/CCA ratio, expressed as median (interquartile range) was $0.71(0.60-0.77)$ in stages I and II, $0.49(0.45-0.57)$ in stages III and IV, and $0.38(0.34-0.47)$ in stages $\mathrm{V}$ and $\mathrm{VI}(P<.001)$. The ICA/CCA ratio was significantly lower in stages $\mathrm{V}$ and $\mathrm{VI}$ than in stages I and II.
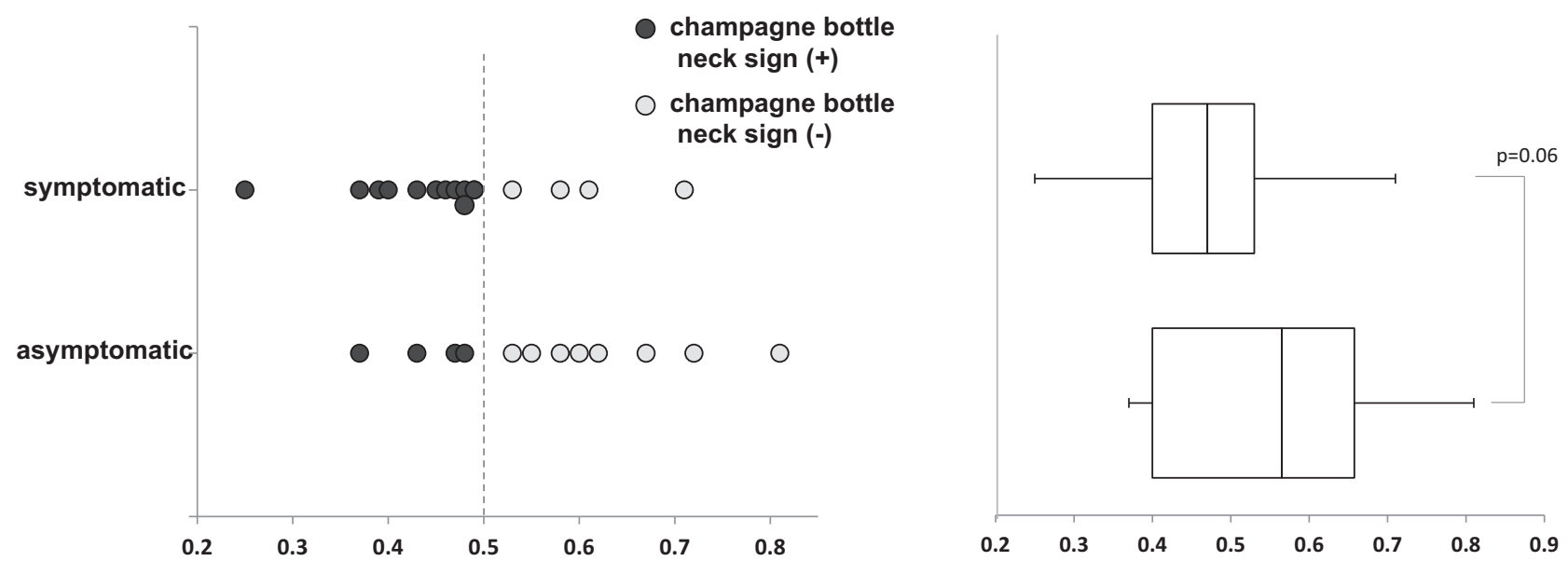

A

ICA/CCA ratio

B

ICA/CCA ratio

FIG 2. Relationships between the ICA/CCA ratio and clinical symptoms. $A$, Symptomatic arteries were more frequently observed in the CBNS-positive group than in the CBNS-negative group (73\% versus 33\%, respectively; Fisher exact test, $P=.06$ ). $B$, The median ICA/CCA ratio in symptomatic arteries tended to be lower than that in asymptomatic arteries $(0.47$ [interquartile range: $0.40-0.53$ ] versus 0.57 [0.47-0.66], respectively; $P=.06$ ).

diagnoses at onset included hemorrhagic stroke $(n=4)$, ischemic stroke $(n=5)$, and transient ischemic attack $(n=4)$. The remaining patient was asymptomatic. Suzuki grades were stage I in 3 arteries, stage II in 2 arteries, stage III in 12 arteries, stage IV in 4 arteries, stage V in 3 arteries, and stage VI in 3 arteries. The ICA/ CCA ratio was obtained by carotid ultrasonography in 20 of 27 arteries and DSA in the remaining 7 arteries. We found CBNS positivity in 15 of the 27 arteries (56\%) (Table).

The ICA/CCA ratio decreased as the clinical stage advanced (Fig 1A). No CBNS was observed in stages I or II, although the CBNS was observed in 5 of 12 stage III arteries (42\%) and in all stage IV-VI arteries. The median ICA/CCA ratio was $0.71(0.60-$ $0.77)$ in stages I and II, $0.49(0.45-0.57)$ in stages III and IV, and $0.38(0.34-0.47)$ in stages V and VI $(P<.001)$ (Fig $1 B)$. With respect to the relationships between the ICA/CCA ratio and clinical symptoms, symptomatic arteries were more frequently ob- served in the CBNS-positive group (Fig 2A). Of 15 CBNS-positive arteries, $11(73 \%)$ were symptomatic (ischemic stroke $[n=5]$, transient ischemic attack $[n=4]$, intracerebral hemorrhage $[n=$ $1]$, and subarachnoid hemorrhage $[n=1])$ and $4(27 \%)$ were asymptomatic. However, of 12 CBNS-negative arteries, 4 (33\%) were symptomatic (ischemic stroke $[n=1]$, transient ischemic attack $[n=1]$, and intracerebral hemorrhage $[n=2])$ and 8 (67\%) were asymptomatic. The ICA/CCA ratio tended to be lower in symptomatic arteries than in asymptomatic arteries (0.47 [0.40-0.53] versus 0.57 [0.47-0.66], respectively; $P=$ .06) (Fig 2B).

In the 6 patients ( 12 arteries) who underwent semiquantitative SPECT with iodine $123 \mathrm{~N}$-isopropyl-p-iodoamphetamine, the ICA/CCA ratio was not related to cerebral perfusion at rest in the MCA territory. However, the CVR in the MCA territory decreased as the ICA/CCA ratio decreased ( $R=0.80, P<.01)$ (Fig 3$)$. Of 9 


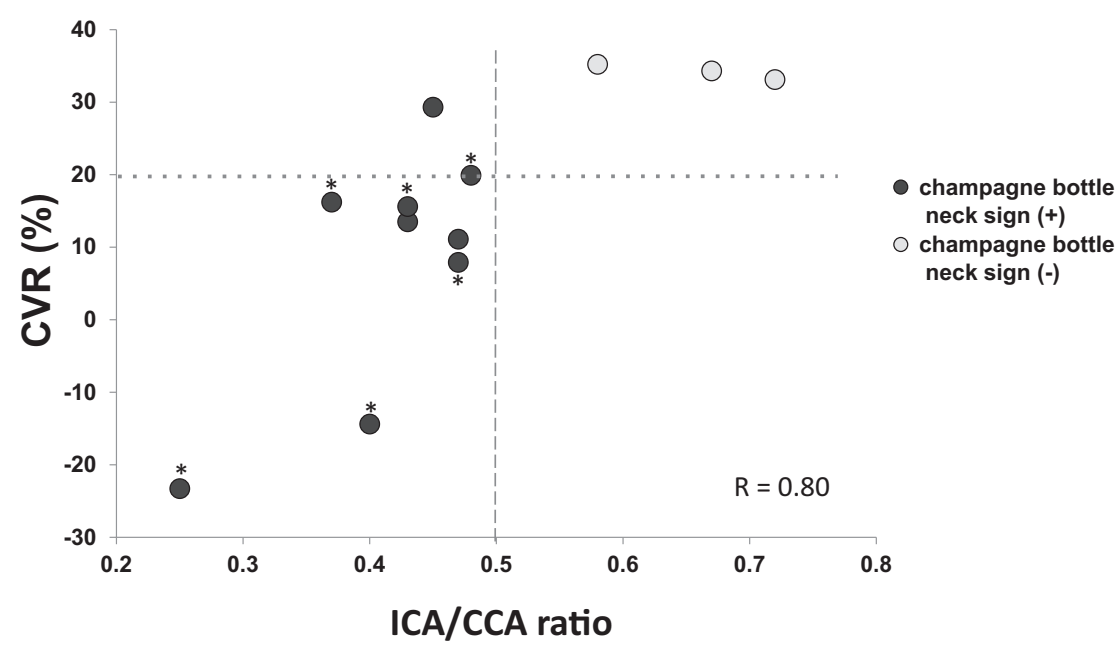

FIG 3. Relationship between the ICA/CCA ratio and CVR to acetazolamide in the MCA territory. The CVR decreased as the ICA/CCA ratio decreased $(R=0.80, P<.01)$. Of 9 arteries with a CBNS, $8(89 \%)$ exhibited a reduced CVR. Symptomatic arteries exhibited both the CBNS and a reduced CVR. Asterisk indicates symptomatic arteries.

carotid ultrasonography

DSA

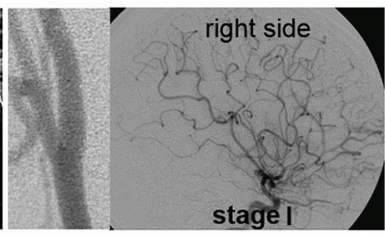

A
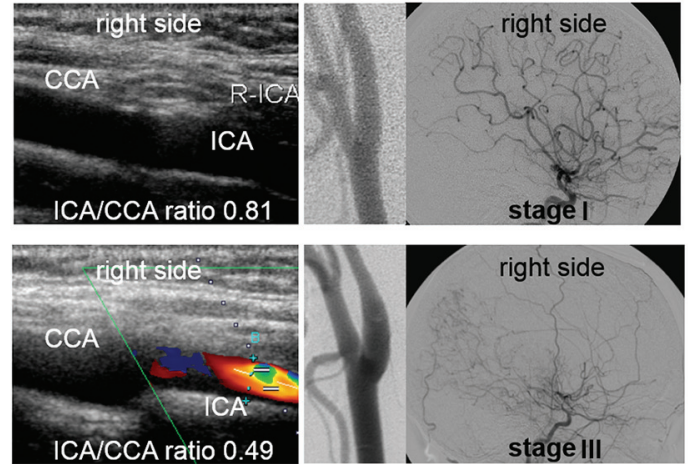

B
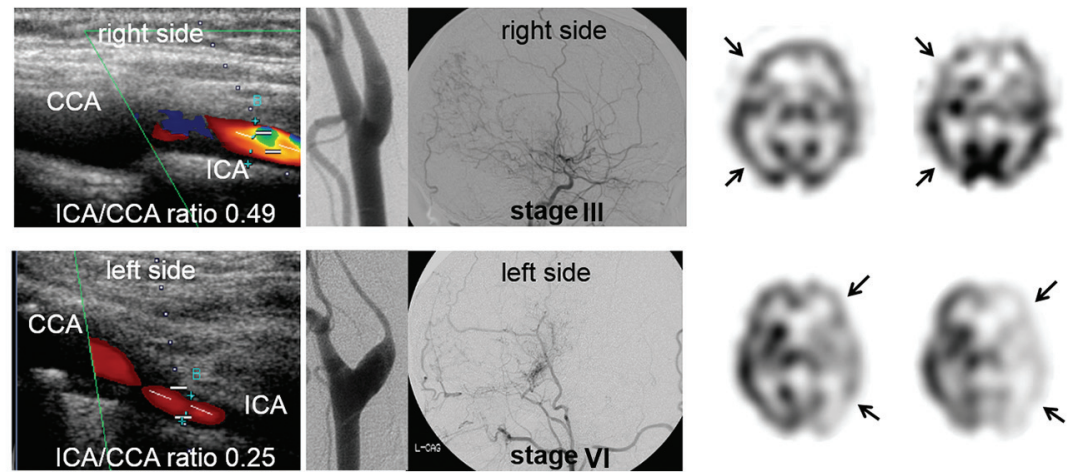

FIG 4. Representative cases. The left, middle, and right columns show the features of carotid ultrasonography, a lateral view of DSA of the carotid and intracranial arteries, and an axial SPECT image from 3 cases: A, A 15-year-old girl had a Suzuki stage I artery on the right (asymptomatic) and a stage II artery on the left (symptomatic). The CBNS was negative on the right by carotid ultrasonography and DSA, with preserved CVR (arrows). B, A 37-year-old woman had a stage III artery on the right (symptomatic) and a stage IV artery on the left (asymptomatic). The CBNS was positive on the right, with a mildly decreased CVR (arrows). C, A 58-year-old woman had a stage II artery on the right (asymptomatic) and a stage VI artery on the left (symptomatic). The CBNS was positive on the left, with a markedly decreased CVR (arrows).

arteries with a CBNS, 8 (89\%) exhibited a reduced CVR. On the other hand, all 3 arteries without a CBNS exhibited a preserved CVR. We observed both the CBNS and a reduced CVR in 6 symptomatic arteries. Of the remaining 6 asymptomatic arteries, only 2 showed both the CBNS and a reduced CVR. Representative cases are shown in Fig 4.

\section{DISCUSSION}

Histopathologic examination of tissue specimens from patients with MMD reveals eccentrically laminated thickening of the in- tracranial major cerebral arteries. ${ }^{10}$ This fibrocellular intimal thickening extends to the extracranial ICA as the disease advances ${ }^{1}$; it is also observed in other arteries, such as the pulmonary, renal, and coronary arteries. ${ }^{11-13}$ At the carotid bifurcation, there is a transitional zone between the elastic ( $>5 \mathrm{~mm}$ proximal to the bifurcation) and muscular $(>15$ mm distal to the bifurcation) portions of the carotid arteries, and the elastic arteries typically have thicker walls (1-2 mm) to tolerate the increased pressure. ${ }^{14,15}$ Therefore, the muscular portion is thought to be more commonly affected, and the narrowing at the transitional zone results in formation of the CBNS. ${ }^{5}$

No systematic studies on the relationship between the presence of the CBNS and the angiographic Suzuki grade have been performed. However, Yasuda et $\mathrm{al}^{4}$ reported that the CBNS might be observed in advanced cases. Our study clearly demonstrated that the CBNS was not observed in the early stage; it began to appear in Suzuki stage III, and all higher Suzuki stages showed the CBNS. The relationships between the CBNS and the presence of clinical symptoms or cerebral hemodynamics also have not been reported. In our study, CBNS-positive arteries were more likely to be symptomatic than CBNS-negative arteries. These findings support the concept that a cerebral hemodynamic state with a reduced CVR is responsible for the occurrence and recurrence of stroke. ${ }^{16,17}$ Previous reports regarding the cerebral hemodynamics of patients with MMD have indicated that cerebral perfusion at rest is not significantly different among the various clinical stages. This is because the blood supply through collateral pathways can compensate for the reduced CBF, even with advancement of the disease. ${ }^{1,18}$ However, CVR tends to diminish as MMD progresses. ${ }^{18}$ We also found that the CBNS was related to impaired CVR, though not to cerebral perfusion. Our results indicate that the risk of stroke can be predicted by the presence of the CBNS.

Although MR imaging is a useful technique with which to evaluate MMD, the extracranial ICAs, unlike the intracranial ICAs, are not always investigated by MRA. An advantage of carotid ultrasonography is that it enables direct evaluation of the extracranial ICAs in real time. Furthermore, it can be noninvasively and repeatedly performed on an outpatient basis or at the AJNR Am J Neuroradiol 37:1898-902 Oct 2016 www.ajnr.org

1901 
bedside. Because of the increasing number of elderly patients with MMD,${ }^{19}$ differential diagnosis between MMD and atherosclerotic occlusive cerebrovascular disease is becoming more important. Although advantages of high-resolution MR wall imaging for the differential diagnosis between MMD and atherosclerosis have been reported, ${ }^{20}$ ultrasonography would be helpful to differentiate these 2 diseases with respect to the morphologically different features of their vessel walls. Especially in young children, sedation is sometimes necessary to perform MR imaging, but not carotid ultrasonography. Therefore, we believe that carotid ultrasonography is useful for screening of MMD and estimation of the clinical stages of MMD. Notably, however, the CBNS is not observed in the early stages of MMD and can also be seen in other diseases such as dissection, fibromuscular dysplasia, and intracranial ICA occlusion of other causes.

This study had some limitations. First, the number of patients was relatively small because we included patients with MMD who underwent DSA. The prevalence of MMD in the general population is low. Furthermore, MMD has recently tended to be more frequently diagnosed by MR imaging or MRA rather than DSA. Second, the arteries of patients with lower Suzuki grades were relatively small. This may suggest bias in the selection process, leading to over-representation of higher Suzuki grades and inflation of the significance of a positive CBNS. Third, a quarter of arteries were evaluated by DSA instead of carotid ultrasonography because of the high position of the carotid bifurcation. However, in the remaining arteries, the ICA/CCA ratio obtained by carotid ultrasonography and that obtained by DSA were not different; therefore, we do not believe that the results were affected.

\section{CONCLUSIONS}

The CBNS is related to Suzuki stage III or higher and impaired CVR with clinical symptoms in patients with MMD. Detection of the CBNS via carotid ultrasonography is useful for not only screening of MMD, but also for determining the clinical and hemodynamic stages of MMD.

\section{ACKNOWLEDGMENTS}

The authors thank the staff of the Departments of Cerebrovascular Disease and Neurosurgery, Japan Labour Health and Welfare Organization, Kyushu Rosai Hospital, Kitakyushu, Japan.

Disclosures: Shuji Arakawa-UNRELATED: Payment for Lectures (including service on Speakers Bureaus): Kyushu Nutrition Welfare University, Comments: Gave lectures on stroke.

\section{REFERENCES}

1. Suzuki J, Takaku A. Cerebrovascular "Moyamoya" disease. Disease showing abnormal net-like vessels in base of brain. Arch Neurol 1969;20:288-99 CrossRef Medline

2. Yang SH, Li B, Wang CC, et al. Angiographic study of Moyamoya disease and histological study in the external carotid artery system. Clin Neurol Neurosurg 1997;99:S61-63 CrossRef Medline

3. Yasaka M, Ogata T, Yasumori K, et al. Bottle neck sign of the proxi- mal portion of the internal carotid artery in Moyamoya disease. $J$ Ultrasound Med 2006;25:1547-52 Medline

4. Yasuda C, Yakusiji Y, Eriguchi M, et al. Usefulness of carotid ultrasonography for the early detection of Moyamoya disease [in Japanese]. Rinsho Shinkeigaku 2007;47:441-43 Medline

5. Shimogawa T, Morioka T, Sayama T, et al. Champagne bottle neck sign in a patient with Moyamoya syndrome. World J Clin Cases 2014; 2:474-77 CrossRef Medline

6. Fukui M. Guidelines for the diagnosis and treatment of spontaneous occlusion of the circle of Willis ('Moyamoya' disease). Research Committee on Spontaneous Occlusion of the Circle of Willis (Moyamoya Disease) of the Ministry of Health and Welfare, Japan. Clin Neurol Neurosurg 1997;99:S238-40 CrossRef Medline

7. Nakagawara J, Takeda R, Suematsu K, et al. Quantification of regional cerebral blood flow and vascular reserve in childhood Moyamoya disease using [123I]IMP-ARG method. Clin Neurol Neurosurg 1997;99:S96-99 CrossRef Medline

8. Saito N, Nakagawara J, Nakamura H, et al. Assessment of cerebral hemodynamics in childhood Moyamoya disease using a quantitative and a semiquantitative IMP-SPECT study. Ann Nucl Med 2004; 18:323-31 CrossRef Medline

9. Honda M, Ezaki Y, Kitagawa N, et al. Quantification of the regional cerebral blood flow and vascular reserve in Moyamoya disease using split-dose iodoamphetamine I 123 single-photon emission computed tomography. Surg Neurol 2006;66:155-59; discussion 159 CrossRef Medline

10. Fukui M, Kono S, Sueishi K, et al. Moyamoya disease. Neuropathology 2000;20:S61-64 Medline

11. Ikeda E. Systemic vascular changes in spontaneous occlusion of the circle of Willis. Stroke 1991;22:1358-62 CrossRef Medline

12. Kaczorowska M, Jozwiak S, Litwin M, et al. Moyamoya disease associated with stenosis of extracranial arteries: a case report and review of the literature. [in Polish] Neurol Neurochir Pol 2005;39:242-46 Medline

13. Lee JH, Youn TJ, Yoon YE, et al. Coronary artery stenosis in Moyamoya disease: tissue characterization by 256-slice multi-detector CT and virtual histology. Circulation 2013;127:2063-65 CrossRef Medline

14. Hori E, Hayashi N, Hamada H, et al. A development of atheromatous plaque is restricted by characteristic arterial wall structure at the carotid bifurcation. Surg Neurol 2008;69:586-90; discussion 590-91 CrossRef Medline

15. Janzen J, Lanzer P, Rothenberger-Janzen K, et al. Variable extension of the transitional zone in the medial structure of carotid artery tripod. Vasa 2001;30:101-06 CrossRef Medline

16. Grubb RL, Derdeyn CP, Fritsch SM, et al. Importance of hemodynamic factors in the prognosis of symptomatic carotid occlusion. JAMA 1998;280:1055-60 CrossRef Medline

17. Yamauchi $H$, Higashi $\mathrm{T}$, Kagawa $\mathrm{S}$, et al. Is misery perfusion still a predictor of stroke in symptomatic major cerebral artery disease? Brain 2012;135:2515-26 CrossRef Medline

18. Kang KH, Kim HS, Kim SY. Quantitative cerebrovascular reserve measured by acetazolamide-challenged dynamic CT perfusion in ischemic adult Moyamoya disease: initial experience with angiographic correlation. AJNR Am J Neuroradiol 2008;29:1487-93 CrossRef Medline

19. Kuriyama S, Kusaka Y, Fujimura M, et al. Prevalence and clinicoepidemiological features of Moyamoya disease in Japan: findings from a nationwide epidemiological survey. Stroke 2008;39:42-47 CrossRef Medline

20. Ryoo S, Cha J, Kim SJ, et al. High-resolution magnetic resonance wall imaging findings of Moyamoya disease. Stroke 2014;45: 2457-60 CrossRef Medline 